\title{
Atomic-Scale Visualization of Ultrafast Bond Breaking in X-Ray-Excited Diamond
}

\author{
Ichiro Inoue, ${ }^{1, *, \dagger}$ Yuka Deguchi, ${ }^{2, \dagger}$ Beata Ziaja $\odot,{ }^{3,4, \$}$ Taito Osaka, ${ }^{1}$ Malik M. Abdullah, ${ }^{3}$ Zoltan Jurek, ${ }^{3}$ \\ Nikita Medvedev, ${ }^{5,6}$ Victor Tkachenko, ${ }^{4,7,3}$ Yuichi Inubushi, ${ }^{8,1}$ Hidetaka Kasai, ${ }^{2,9}$ Kenji Tamasaku, ${ }^{1}$ \\ Toru Hara, ${ }^{1}$ Eiji Nishibori ${ }^{2,9,8}$ and Makina Yabashi ${ }^{1,8}$ \\ ${ }^{1}$ RIKEN SPring-8 Center, 1-1-1 Kouto, Sayo, Hyogo 679-5148, Japan \\ ${ }^{2}$ Graduate School of Pure and Applied Sciences, University of Tsukuba, Tsukuba, Ibaraki 305-8571, Japan \\ ${ }^{3}$ Center of Free-Electron Laser Science, Deutsches Elektronen-Synchrotron, Notkestraße 85, 22607, Germany \\ ${ }^{4}$ Institute of Nuclear Physics, Polish Academy of Sciences, Radzikowskiego 152, 31-342 Krakow, Poland \\ ${ }^{5}$ Institute of Physics, Czech Academy of Sciences, Na Slovance 2, Prague 8, 18221, Czech Republic \\ ${ }^{6}$ Institute of Plasma Physics, Czech Academy of Sciences, Za Slovankou 3, 18200 Prague 8, Czech Republic \\ ${ }^{7}$ European XFEL GmbH, Holzkoppel 4, 22869 Schenefeld, Germany \\ ${ }^{8}$ Japan Synchrotron Radiation Research Institute, Kouto 1-1-1, Sayo, Hyogo 679-5198, Japan \\ ${ }^{9}$ Faculty of Pure and Applied Sciences and Tsukuba Research Center for Energy Materials Science, University of Tsukuba, \\ Tsukuba, Ibaraki 305-8571, Japan
}

(Received 1 June 2020; revised 9 December 2020; accepted 23 January 2021; published 19 March 2021)

\begin{abstract}
Ultrafast changes of charge density distribution in diamond after irradiation with an intense x-ray pulse (photon energy, $7.8 \mathrm{keV}$; pulse duration, $6 \mathrm{fs}$; intensity, $3 \times 10^{19} \mathrm{~W} / \mathrm{cm}^{2}$ ) have been visualized with the $\mathrm{x}$-ray pump-X-ray probe technique. The measurement reveals that covalent bonds in diamond are broken and the electron distribution around each atom becomes almost isotropic within $\sim 5 \mathrm{fs}$ after the intensity maximum of the x-ray pump pulse. The 15 fs time delay observed between the bond breaking and atomic disordering indicates nonisothermality of electron and lattice subsystems on this timescale. From these observations and simulation results, we interpret that the x-ray-induced change of the interatomic potential drives the ultrafast atomic disordering underway to the following nonthermal melting.
\end{abstract}

DOI: 10.1103/PhysRevLett.126.117403

Since their discovery in 1895 , $x$ rays have become established as an invaluable probe for gaining an atomic insight into the structure of matter through various kinds of interaction processes, such as scattering, absorption, and emission of photoelectrons and fluorescence. These interactions were usually weak with the previous x-ray sources, and therefore it was assumed that x-ray irradiation did not modify matter. This situation has been totally changed by the recent advent of $\mathrm{x}$-ray free-electron lasers (XFELs) [1,2], which can generate brilliant femtosecond $\mathrm{x}$-ray pulses.

When an XFEL pulse irradiates matter, photoionization and emission of Auger electrons occur almost simultaneously during or shortly after the irradiation with the pulse. The emitted electrons collide with bound electrons in the surrounding atoms and strip outer shell electrons, triggering a cascade of collisional ionization [3], and thereby a massive number of electrons are excited almost at once. For example, a numerical simulation [4] predicts that an XFEL pulse with an intensity of $\sim 10^{19} \mathrm{~W} / \mathrm{cm}^{2}$ [5] may excite $\sim 30 \%$ of valence electrons to the conduction band for diamond, causing irreversible atomic disordering. A deep understanding of transient XFEL interaction with matter is essential not only because of fundamental interest but for analyzing experiments with intense XFEL pulses and designing optical devices applicable to future cavitybased XFELs [6,7].

So far, time-resolved description of XFEL interaction with matter has been relying on theoretical modeling, validated only in part by time-integrated measurements of charge states of ions $[8,9,10]$ and emitted fluorescence $[11,12]$. A recently developed $\mathrm{x}$-ray pump-x-ray probe diffraction technique using a double XFEL pulse is promising for capturing the transient interaction processes. A few pioneering groups applied this technique to benchmarking samples (diamond [13], silicon [14], and xenon clusters [15]) and revealed that the XFEL pulse causes atomic displacements on the femtosecond timescale. These experiments, however, do not answer fundamental questions, such as what physical mechanism leads to ultrafast atomic displacement and how electron excitation proceeds with time, because a limited number of the measured diffraction peaks (one or two) made it difficult to discuss detailed structural information.

We here demonstrate an atomic-scale time-resolved visualization of XFEL-matter interaction. By employing the unique capability of SACLA [16] that can generate XFEL pulses with the shortest wavelength among the similar facilities, several probe diffraction peaks of diamond were measured in the $\mathrm{x}$-ray pump-x-ray probe 
experiment. The charge density distribution was determined from the relative intensity of the probe diffraction peaks by the multipole electron-density modeling [17]. From the visualized charge density distribution, the mechanisms of femtosecond atomic disordering previously reported in $[13,14,15]$ are discussed.

Figure 1(a) shows a schematic illustration of the experiment at SACLA beamline 3 [18]. The XFEL source was operated in the two-color double pulse mode [19] to generate $7.8-\mathrm{keV}$ pump and $11.5-\mathrm{keV}$ probe pulses with pulse durations of $6 \mathrm{fs}$ (FWHM) [20]. The delay of the probe pulse with respect to the pump was controlled at the accuracy of less than $1 \%$ of the delay time by using a magnetic chicane [19]. The double pulse was focused to an FWHM of $200 \mathrm{~nm}$ diameter by an x-ray mirror system [21]. By using three multiport charge-coupled device (MPCCD) detectors [22], diffraction peaks $(111,220,311,400,331$ reflections of the probe and 220 reflection of the pump) from a nanocrystal diamond film with $16 \mu \mathrm{m}$ thickness [23] were measured with the different time delays of the probe pulses. The shot-by-shot pulse energy was determined by an inline spectrometer [24], and $~ 500$ pulses with the specific pulse energies of the pump $(69 \pm 10 \mu \mathrm{J})$ and the probe pulses $(29 \pm 6 \mu \mathrm{J}$ ) (corresponding to $\sim 3 \times 10^{19} \mathrm{~W} / \mathrm{cm}^{2}$ and $\sim 1 \times 10^{19} \mathrm{~W} / \mathrm{cm}^{2}$, respectively) were extracted for each delay and the corresponding diffraction images were used for the following analysis. For the purpose of calibration, we also measured diffraction intensity of undamaged diamond by reducing $\mathrm{x}$-ray fluence to less than the damage threshold using a silicon attenuator.
Figures 1(b) and 1(c) show the diffraction intensity profiles for the two shortest delay times ( 0.5 and $5 \mathrm{fs})$ and with longer delay times added, respectively. For better visibility, the profiles normalized by the peak intensity of probe 111 reflection are shown in Fig. 1(b), while those in Fig. 1(c) are rescaled in such a way that the baselines of probe 111 reflection become the same degree. Interestingly, the peak positions of all reflections were almost the same for different delay conditions, indicating that lattice expansion due to ablation was insignificant at the measured timescale. The normalized probe diffraction intensity between the shortest two delay times was slightly different $(\sim 10 \%)$ for 220, 400, and 331 reflections [Fig. 1(b)]. To check the significance of this small difference, we separated all diffraction images into three groups for each delay condition and evaluated the probe diffraction intensity [as an example, the intensity profiles calculated with the separated data set for delay time of $0.5 \mathrm{fs}$ are shown in the insets of Fig. 1(b)]. The relative difference between the normalized diffraction intensity calculated with the separated data set and that with the full data set was less than 5\% for all reflections. Thus, the uncertainty of the measured probe diffraction intensity when all diffraction images were used for the analysis can be estimated to $5 \% / \sqrt{3}=3 \%$ at most. Therefore, the difference in the probe diffraction intensity in Fig. 1(b) is statistically significant. With increasing delay times, the probe diffraction intensity rapidly decreased, especially for the higher reflection indices [Fig. 1(c)].

Integrated diffraction intensity for each reflection was evaluated by the profile fitting and the Rietveld refinement.

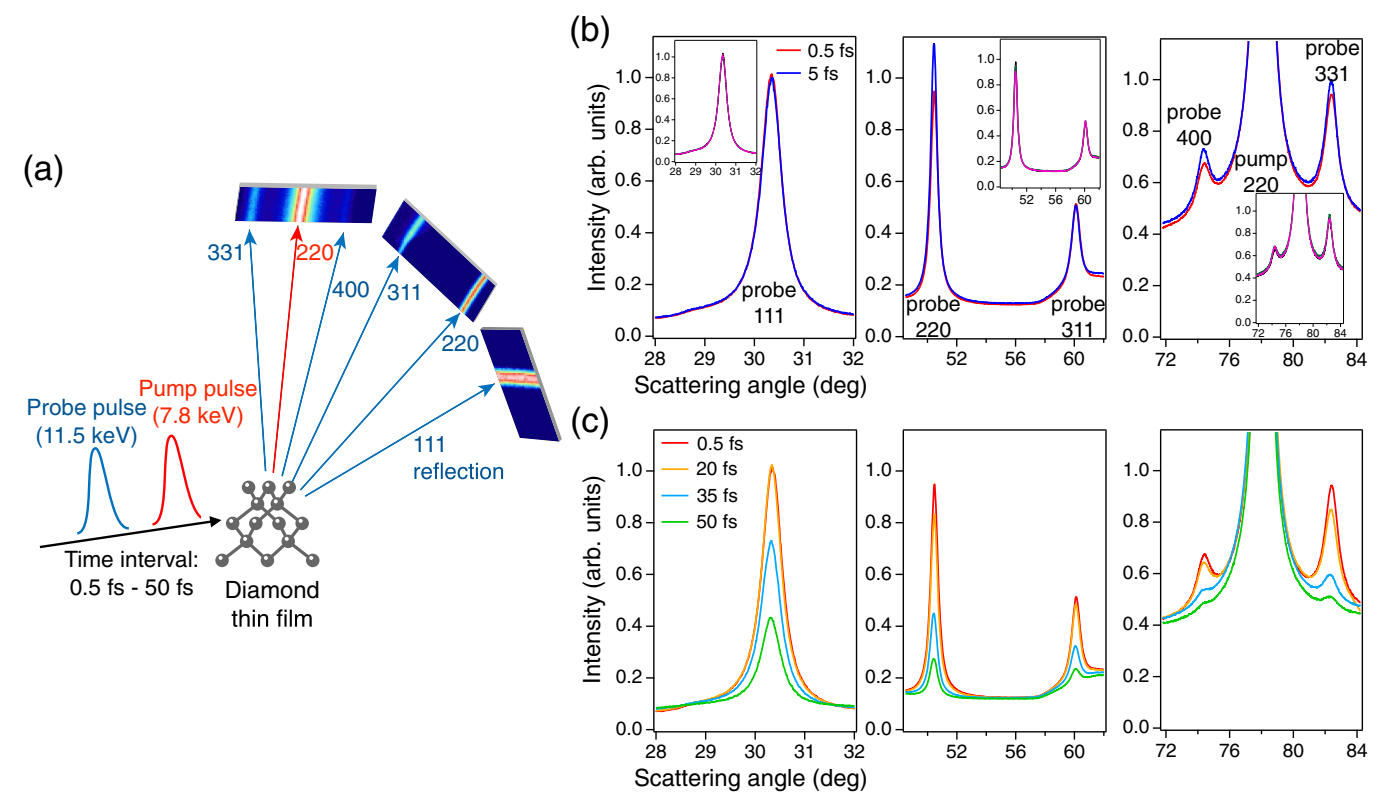

FIG. 1. (a) A schematic illustration of the experiment. The focused double XFEL pulse irradiated a diamond film. The shot-by-shot diffraction images were measured using three MPCCD detectors. (b) Diffraction intensity profiles for delay times of 0.5 and 5 fs, which are normalized by peak intensity of probe 111 reflection. The insets show those for delay time of 0.5 fs calculated with separated data set (see the main text). (c) Diffraction intensity profiles for delay times of $0.5,20,35$, and 50 fs. 
By dividing the square of structure factors of diamond measured with synchrotron radiation [25] by the integrated diffraction intensity of undamaged diamond, a geometrical correction factor was determined for each reflection. Then, the integrated diffraction intensity for x-ray-excited diamond was multiplied by the correction factor and used for structure refinement.

We determined the charge density distribution in diamond by the multipole electron-density modeling in the Hansen-Coppens formalism [26]. To simplify the model, the experimental data were analyzed under the following two assumptions. First, it was assumed that the number of core holes was negligibly small and the charge density distribution of the innershell electrons was fairly the same as the one for undamaged diamond at $300 \mathrm{~K}$. The second assumption was that the $\mathrm{x}$-ray-induced atomic displacements were independent and random with a mean of zero. Multipole refinement was performed by using XD2016 [27]. In the analysis, the relativistic scattering factor in the Su-Coppens-Macchi scattering databank $[28,29]$ was adopted and electron configuration of $1 s^{2}, 2 s^{2}, 2 p^{2}$ was selected. In this model, the electron-density distribution is simply described by the following seven parameters: the scale factor $s$, the isotropic displacement $U_{\text {iso }}$, the radial expansion and contraction parameters for the spherical $(\kappa)$ and aspherical $\left(\kappa^{\prime}\right)$ parts, the octupole $\left(\mathrm{O}_{2}-\right)$, and the hexadecapoles $\left(\mathrm{HO}\right.$ and $\mathrm{H} 4+$ ). Here, $\kappa$ and $\mathrm{O}_{2}-$ are the main components that determine valence charge density distribution in diamond [25]. Also, $\mathrm{H} 4+$ is constrained to $H 0$ by $H 4+=(0.74048) H 0$ due to the symmetry of diamond. The multipole refinement was performed using $s, U_{\text {iso }}, \kappa$, and $O 2-$, while the other three parameters $\left(\kappa^{\prime}\right.$, $H 0, H 4)$ were set to be the same as those for undamaged diamond [25]. The refinement of the modeling resulted in excellent crystal reliability factors ( $R$ factors) $[R$ factors: $3.0 \%$ (delay time of $0.5 \mathrm{fs}$ ), $5.2 \%$ ( $5 \mathrm{fs}$ ), $8.9 \%$ (10 fs), $5.5 \%$ (20 fs), 3.0\% (35 fs), and 3.3\% (50 fs)], which are comparable to the previous charge density studies of diamond [25,30-37].

Figure 2(a) shows the determined valence charge density distribution for the (110) plane. For reference, the one at the undamaged state is also shown. Interestingly, even for the shortest delay time ( $0.5 \mathrm{fs})$, the charge density distribution was different from that at the undamaged state. Here, we emphasize that $\mathrm{x}$-ray diffraction techniques, including the present method, determine the ensemble-averaged structure. However, considering: (i) the short time needed by electrons to thermalize after the XFEL irradiation-simulation of secondary ionization processes with the XCASCADE code [38] predicts that electron cascade continues until $\sim 25$ fs after the pump pulse, and (ii) the fast electron spreading (on $100 \mathrm{~nm}$ distances within $25 \mathrm{fs}$, for details see [39]), we find that the charge density distribution may differ from atom to atom only during or shortly after (a)
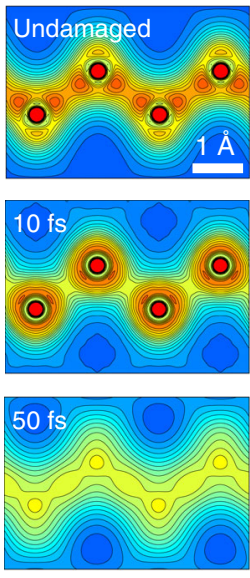

$0.0 \underset{\text { end }}{0.3} 2.0 \mathrm{e}^{-3} \AA^{-3}$
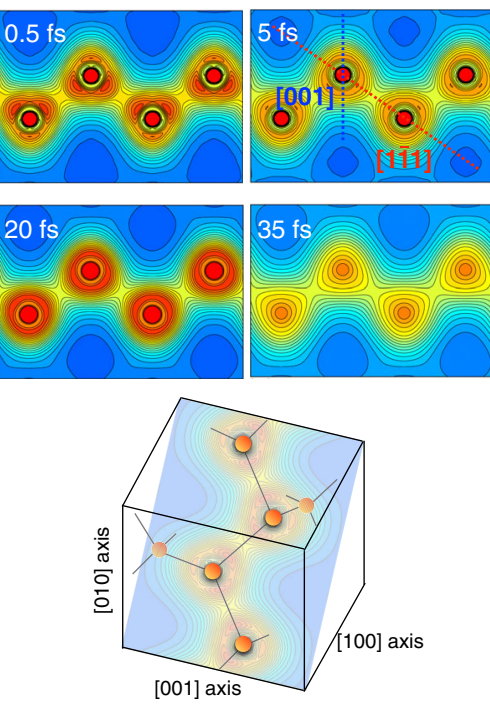

(b)

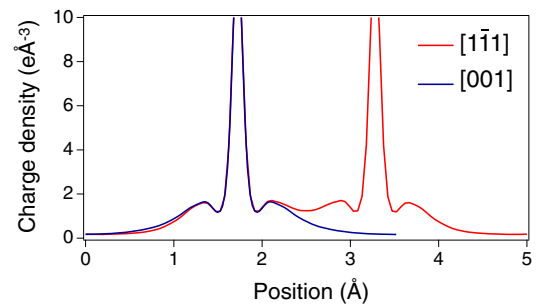

(c)

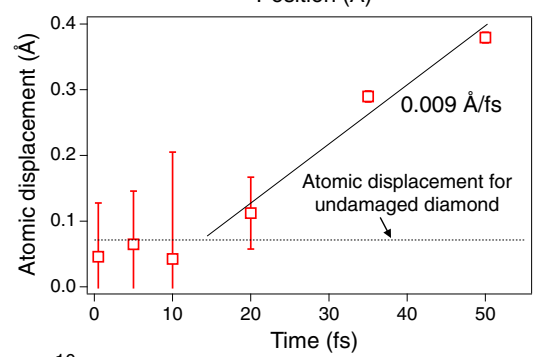

(d)

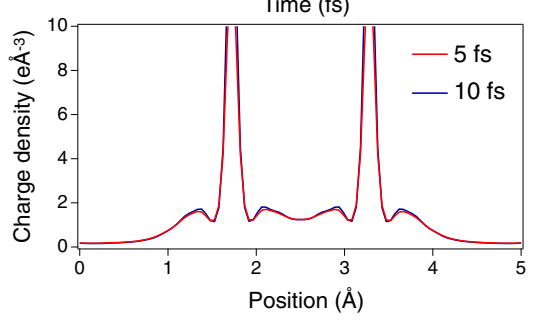

FIG. 2. (a) Valence charge density distribution of diamond for the (110) plane at different delay times. The contour lines are drawn from 0.1 to $2.0 e \AA^{-3}$ with $0.1 e \AA^{-3}$ step width $\left(e=1.6 \times 10^{-19} \mathrm{C}\right.$ is the electron charge). (b) Line profiles of the valence charge density along [1 11 ] and [001] directions [see Fig. 2(a)] for delay time of 5 fs. (c) Root-mean-square atomic displacement after irradiation with the pump pulse. (d) Line profiles of the valence charge density along [111] direction for delay times of 5 and 10 fs. 
the XFEL pulse. Although such electron inhomogeneity cannot be evaluated solely from diffraction intensity, it can safely be said that charge density distribution temporally changed within the time scale of XFEL pulse duration from the difference in the determined charge density distribution for 0.5 fs delay and the undamaged state. Spectroscopic techniques, such as x-ray Thomson scattering [44], could be useful for investigating the electron inhomogeneity in more detail.

For delay times longer than $5 \mathrm{fs}$, the electron distribution surrounding each atom became almost isotropic, indicating that the covalent bonds were broken. This result is more clearly shown in Fig. 2(b), in which the valence charge density along [1111] and [001] directions around a carbon atom is plotted. The isotropic charge density distribution implies that the electron excitation induced by the pump pulse weakened the interaction between neighboring atoms. Because of the weak interatomic interaction, each atom is expected to behave like an isolated atom and start to move from its original position after the bond breaking. In fact, the root-mean-square atomic displacement started to linearly increase with time at the rate of $9 \times 10^{-3} \AA / \mathrm{fs}$ after the bond breaking [Fig. 2(c)]. This rate was comparable to the velocity of an isolated carbon atom at room temperature $\left(\sqrt{3 k_{B} T / M}=8 \times 10^{-3} \AA / \mathrm{fs}\right.$, where $k_{B}$ is the Boltzmann constant, $T=300 \mathrm{~K}$, and $M$ is the mass of a carbon atom). It is noteworthy that the charge density distribution for delay times of 5 and 10 fs [Figs. 2(a) and 2(d)] was almost the same, indicating that significant electron excitation to the conduction band occurred by $\sim 5$ fs after the intensity maximum of the pump pulse. Because the onset of the atomic displacement ( $20 \mathrm{fs})$ was delayed with the electron excitation, the momentum of each atom would not change upon the irradiation with the pump pulse in accordance with the Franck-Condon principle. Therefore, (i) the similarity between the increase rate of the atomic displacement and the velocity of the isolated atom and (ii) the linear time dependence of the atomic displacement indicate that the electron excitation made the potential energy surface almost flat and drove the inertial motion of atoms. The observed melting process can be considered as an x-ray analog to nonthermal melting in semiconductors induced by femtosecond optical laser pulse [45-51], where excitation of valence electrons to conduction band modifies interatomic potential and triggers femtosecond solid-toliquid transition. Notably, linear time dependence of the atomic displacement was also observed in the nonthermal melting in semiconductors $[45,46]$.

To verify our interpretation of the experimental results, we applied the recently developed code XTANT (x-rayinduced thermal and nonthermal transitions) [42] for modeling x-ray-excited diamond at the experimental conditions (see [39] for details). In the present simulation, we took into account the effect of the spatial Gaussian profile of the $\mathrm{x}$-ray pulses as well as the escape of energetic electrons from the $\mathrm{x}$-ray focus [41].

Figure 3(a) shows the average charge density of valence and conduction band electrons after the irradiation with the (a)

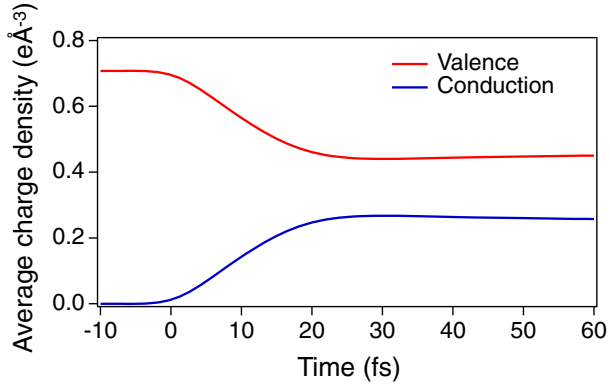

(b)

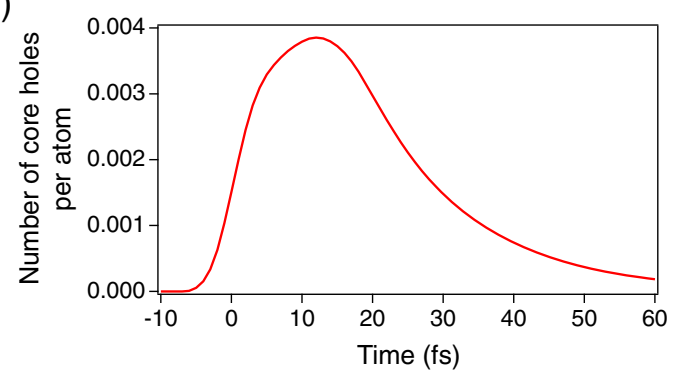

(c)

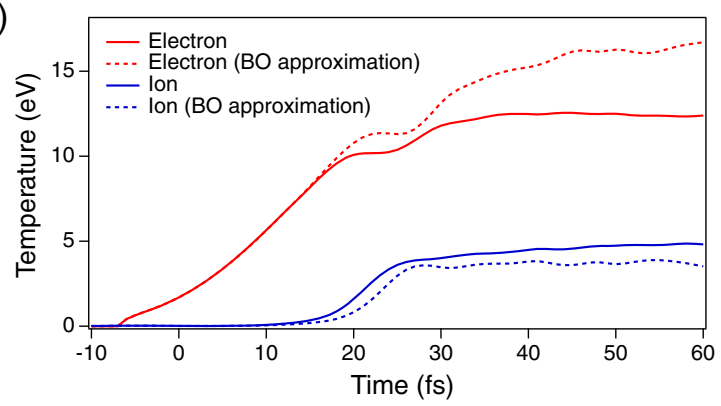

FIG. 3. Femtosecond excitations of electrons and ions in x-ray-irradiated diamond simulated with XTANT code. (a) Average charge density of valence and conduction band electrons after the irradiation with the pump pulse. Time zero in the horizontal axis corresponds to the intensity maximum of the pump pulse. (b) Number of core holes per atom created after the irradiation with the pump pulse. (c) Ion and electron temperatures after the irradiation with the pump pulse. The simulation results obtained with the Born-Oppenheimer approximation are also shown for comparison. 
pump pulse. The simulation predicts that excitation of valence electrons to the conduction band takes place immediately after the irradiation with the pump pulse. This ultrafast electron excitation and its timescale are consistent with the bond breaking observed around 5 fs after the pump pulse [Fig. 2(a)]. In contrast to the massive excitation of the valence electrons, the fraction of core-hole atoms is quite small (much less than 1\%) [Fig. 3(b)]. This supports our earlier assumption made that the $\mathrm{x}$-ray irradiation does not change the charge density distribution of the innershell electron on the femtosecond timescale.

Figure 3(c) shows simulated electron and ion temperatures. The electron temperature is calculated only for thermalized electrons in the valence and in the bottom of the conduction band. The electron temperature is higher than the ion temperature on the simulated timescales, indicating that the system is not yet thermally equilibrated. The onset of the ion temperature increase is $\sim 20 \mathrm{fs}$, which agrees well with the experimental observation [Fig. 2(c)]. In contrast, the electron temperature starts to rise immediately after the irradiation with the pump pulse. This result confirms that ions remained "cold" before the onset of atomic disordering, and implies that the electron-ion thermal interaction did not drive the melting of diamond observed in the present experiment. Therefore, we can conclude that the ultrafast atomic displacement observed in the experiment was due to the nonthermal changes of the potential energy surface and not due to the electron-ion energy exchange.

To justify this statement, we also performed a simulation within the Born-Oppenheimer (BO) approximation that naturally excludes electron-ion coupling, and calculated the respective electron and ion temperature after the irradiation with the pump pulse. The BO results are fairly close to the original simulation results that include electron-ion coupling [Fig. 3(c)]. This confirms a negligible contribution of electron-ion coupling on the simulated timescales and proves that the ultrafast melting of diamond is of a nonthermal origin.

In summary, the charge density distribution of diamond after irradiation with an XFEL pulse was visualized at atomic resolution through $\mathrm{x}$-ray pump-X-ray probe experiment. It was found that the covalent bonds were broken within $\sim 5$ fs after the XFEL irradiation and that inertial atomic motion was caused by the change of interatomic potential. The x-ray-induced nonthermal melting should be ubiquitous for many experiments with intense XFELs, such as single-particle imaging [52], protein nanocrystallography [53], and generation of warm-dense-matter and plasma in the high-energy-density regime [11]. Particularly, the finding of this study will contribute to developing methodologies for structure determination with intense XFEL pulses, as in this regime, the X-ray-induced damage, first electronic and later structural, occurring during the irradiation should be carefully treated $[54,55]$.
We acknowledge Prof. Hitoki Yoneda for motivating this research and valuable advice, and Dr. Vladimir Lipp for helpful discussions. This study was partially supported by JSPS KAKENHI (Grants No. 17K14137 and No. 19K20604) and the Czech Ministry of Education, Youth and Sports, Czech Republic (Grants No. LTT17015 and No. LM2015083). The experiment was performed with the approval of the Japan Synchrotron Radiation Research Institute (Proposal No. 2018A8040).

*inoue@spring8.or.jp

These authors contributed equally to this Letter.

tbeata.ziaja-motyka@cfel.de

${ }^{\S}$ nishibori.eiji.ga@u.tsukuba.ac.jp

[1] B. W. J. McNeil and N. R. Thompson, Nat. Photonics 4, 814 (2010).

[2] E. L. Saldin, E. A. Schneidmiller, and M. V. Yurkov, The Physics of Free Electron Lasers (Springer, Berlin, 1999).

[3] B. Ziaja, R. A. London, and J. Hajdu, J. Appl. Phys. 97, 064905 (2005).

[4] N. Medvedev and B. Ziaja, Sci. Rep. 8, 5284 (2018).

[5] H. Mimura, H. Yumoto et al., Nat. Commun. 5, 3539 (2014)

[6] K. J. Kim, Y. Shvydko, and S. Reiche, Phys. Rev. Lett. 100, 244802 (2008).

[7] A. Halavanau, A. Benediktovitch, A. A. Lutman, D. DePonte, D. Cocco, N. Rohringer, U. Bergmann, and C. Pellegrini, Proc. Natl. Acad. Sci. U.S.A. 117, 15511 (2020).

[8] L. Young, E. P. Kanter et al., Nature (London) 466, 56 (2010).

[9] H. Fukuzawa and S.-K. Son, K. Motomura, S. Mondal, K. Nagaya et al., Phys. Rev. Lett. 110, 173005 (2013).

[10] B. F. Murphy, T. Osipov et al., Nat. Commun. 5, 4281 (2014).

[11] S. M. Vinko, O. Ciricosta et al., Nature (London) 482, 59 (2012).

[12] S. M. Vinko, O. Ciricosta et al., Nat. Commun. 6, 6397 (2015).

[13] I. Inoue, Y. Inubushi et al., Proc. Natl. Acad. Sci. U.S.A. 113, 1492 (2016).

[14] T. Pardini, J. Alameda, A. Aquila, and S. Boutet, T. Deckeret al., Phys. Rev. Lett. 120, 265701 (2018).

[15] K. R. Ferguson, M. Bucher et al., Sci. Adv. 2, e1500837 (2016).

[16] T. Ishikawa, H. Aoyagi et al., Nat. Photonics 6, 540 (2012).

[17] P. Coppens, X-ray Charge Densities and Chemical Bonding (Oxford University Press, Oxford, 1997).

[18] K. Tono, T. Togashi et al., New. J. Phys. 15, 083035 (2013).

[19] T. Hara, Y. Inubushi et al., Nat. Commun. 4, 2919 (2013).

[20] I. Inoue, T. Hara, Y. Inubushi, K. Tono, T. Inagaki, T. Katayama, Y. Amemiya, H. Tanaka, and M. Yabashi, Phys. Rev. Accel. Beams 21, 080704 (2018).

[21] H. Yumoto, Y. Inubushi et al., Appl. Sci. 10, 2611 (2020).

[22] T. Kameshima, S. Ono et al., Rev. Sci. Instrum. 85, 033110 (2014).

[23] K. Tono, T. Kudo, M. Yabashi, T. Tachibana, Y. Feng, D. Fritz, J. Hastings, and T. Ishikawa, Rev. Sci. Instrum. 82, 023108 (2011). 
[24] K. Tamasaku, Y. Inubushi, I. Inoue, K. Tono, M. Yabashi, and T. Ishikawa, J. Synchrotron Radiat. 23, 331 (2016).

[25] Y. Deguchi and E. Nishibori, Acta. Crystallogr. Sect. B 74, 651 (2018).

[26] N. K. Hansen and P. Coppens, Acta. Crystallogr. Sect. A 34, 909 (1978).

[27] A. Volkov, P. Macchi et al., XD2016-A Computer Program Package for Multipole Refinement, Topological Analysis of Charge Densities and Evaluation of Intermolecular Energies from Experimental and Theoretical Structure Factors, http://www.chem.gla.ac.uk/ louis/xd-home/ (2016).

[28] Z. Su and P. Coppens, Acta Crystallogr. Sect. A 54, 646 (1998).

[29] P. Macchi and P. Coppens, Acta Crystallogr. Sect. A 57, 656 (2001).

[30] R. F. Stewart, J. Chem. Phys. 58, 4430 (1973).

[31] M. A. Spackman, Acta Crystallogr. Sect. A 47, 420 (1991).

[32] K. Yamamoto, Y. Takahashi, K. Ohshima, F. P. Okamura, and K. Yukino, Acta Crystallogr. Sect. A 52, 606 (1996).

[33] Y. A. Abramov and F. P. Okamura, Acta Crystallogr. Sect. A 53, 187 (1997).

[34] E. Nishibori, E. Sunaoshi, A. Yoshida, S. Aoyagi, K. Kato, M. Takata, and M. Sakata, Acta Crystallogr. Sect. A 63, 43 (2007).

[35] H. Svendsen, J. Overgaard, R. Busselez, B. Arnaud, P. Rabiller, A. Kurita, E. Nishibori, M. Sakata, M. Takata, and B. B. Iversen, Acta Crystallogr. Sect. A 66, 458 (2010).

[36] A. Fischer, D. Tiana et al., J. Phys. Chem. A115, 13061 (2011).

[37] N. Bindzus, T. Straaso, N. Wahlberg, J. Becker, L. Bjerg, N. Lock, A.-C. Dippel, and B. B. Iversen, Acta Crystallogr. Sect. A 70, 39 (2014).

[38] N. Medvedev, Appl. Phys. B 118, 417 (2015).
[39] See Supplemental Material at http://link.aps.org/ supplemental/10.1103/PhysRevLett.126.117403 for details about the simulation of XFEL-diamond interaction, which includes Refs. [38,40-43].

[40] V. Lipp, N. Medvedev, and B. Ziaja, Proc. SPIE Int. Soc. Opt. Eng. 10239, 102360H (2017).

[41] R. Follath, T. Koyama, V. Lipp, N. Medvedev, K. Tono, H. Ohashi, L. Patthey, M. Yabashi, and B. Ziaja, Sci. Rep. 9, 2029 (2019).

[42] N. Medvedev, H. O. Jeschke, and B. Ziaja, New. J. Phys. 15, 015016 (2013).

[43] N. Medvedev, Z. Li, V. Tkachenko, and B. Ziaja, Phys. Rev. B 95, 014309 (2017).

[44] S. H. Glenzer and R. Redmer, Rev. Mod. Phys. 81, 1625 (2009).

[45] A. M. Lindenberg, J. Larsson et al., Science 308, 392 (2005).

[46] K. J. Gaffney, A. M. Lindenberg et al., Phys. Rev. Lett. 95, 125701 (2005).

[47] S. K. Sundaram and E. Mazur, Nat. Mater. 1, 217 (2002).

[48] C. V. Shank, R. Yen, and C. Hirlimann, Phys. Rev. Lett. 51, 900 (1983).

[49] P. Stampfli and K. H. Bennemann, Phys. Rev. B 49, 7299 (1994).

[50] K. Sokolowski-Tinten, J. Bialkowski, and D. vonder Linde, Phys. Rev. B 51, 14186 (1995).

[51] A. Rousse et al., Nature (London) 410, 65 (2001).

[52] R. Neutze, R. Wouts, D. van der Spoel, E. Weckert, and J. Hajdu, Nature (London) 406, 752 (2000).

[53] H. N. Chapman, P. Fromme et al., Nature (London) 470, 73 (2011).

[54] A. Barty, C. Caleman et al., Nat. Photonics 6, 35 (2012).

[55] H. M. Quiney and K. A. Nugent, Nat. Phys. 7, 142 (2011). 